\title{
Research on the detection and early warning technology of harmful marine organisms in the water intake of nuclear power plant by 3D image sonar
}

\author{
Jianfei Zhang ${ }^{1, *}$, Zhongpeng $\mathrm{Wu}^{1}$, Chong $\mathrm{An}^{1}$ \\ ${ }^{1}$ Dalian measurement and Control Technology Research Institute, NO. 16, BinhaiStreet,Zhongshan District, \\ Dalian,Liaoning,116013, P. R. China Jianfei_zhang760@163.com
}

\begin{abstract}
In view of the frequent water intake blockage caused by marine biological invasion in many nuclear power plants, which affects the safety of cold source in nuclear power plants, a defense system in depth including marine biological identification, detection, early warning and hierarchical response is established through investigation and analysis of the causes of the event. Underwater acoustic highresolution multi beam detection method is used to detect jellyfish, hairy shrimp and other marine organisms, The echo characteristics are obtained, the distribution density in sensitive sea area is analyzed, and the effective early warning judgment is given. Among them, detection and early warning is the front-end disposal measure, which can provide accurate and timely marine biological information for the subsequent hierarchical response. At the same time, it can replace the underwater inspection work of divers,reduce the risk of divers,reduce the risk of industrial safety, and improve the reliability of cold source.
\end{abstract}

\section{Introduction}

Some data show that with the change of climate, marine and river environmental conditions, the frequent occurrence of water intake blockage events indicates that the nuclear power plants have insufficient risk prediction of marine organisms in the water intake area, the monitoring and early warning mechanism of marine organisms is not perfect, and the response measures are not targeted. Since 2011, the safe and reliable operation of circulating water system has been affected by jellyfish, Phaeocystis, sediment, small fish and shrimp in many nuclear power units at home and abroad, which blocked the cooling water pipe from the turbine of the power plant, resulting in the power plant having to shut down the reactor, seriously affecting the safety of the unit. Since 2016, the national nuclear safety administration has reported that dozens of incidents occurred in domestic nuclear power plants, which affected the safety of water intake due to marine organisms or foreign matters blocking the water intake system. ([1]-[3])Ten typical incidents of marine organisms affecting the safety of water intake of nuclear power plants were disclosed. Under the risk of frequent marine biological invasion, the problem of cold source support of coastal nuclear power plants is becoming increasingly prominent. How to solve the cold source problem of nuclear power plants from the source has become an urgent task. Therefore, the establishment of marine biological detection and early warning mechanism has become the top priority.

\section{Design and development of autonomo us monitoring and early warning syste $m$ for marine organisms in nuclear pow er plant}

The automatic monitoring and early warning system for marine organisms in nuclear power plant uses highresolution multi beam sonar as sensing means, and uses the feature analysis and statistics module to analyze and process the obtained marine organism image information and identify the features, so as to obtain the density distribution of jellyfish, hairy shrimp and other marine organisms in the monitoring area in real time, and give an alarm when the threshold is exceeded.

\subsection{Data processing and analysis of interception network + kelp echo}

The autonomous monitoring and early warning system for marine organisms of nuclear power includes shore based monitoring center and underwater monitoring platform. The shore based detection center is composed of early warning processing unit and database; The underwater monitoring platform is composed of cruise monitoring platform and several fixed monitoring platforms (configured according to the monitoring water area width of the cold source water intake of the nuclear power plant). The fixed monitoring platform is mainly composed of controllable lifting platform, highresolution multi beam sonar, underwater camera,

*Jianfei Zhang: Jianfei_zhang760@163,.com 
hydrological and water quality monitoring module and wireless transmission module; Cruise monitoring platform is mainly composed of autonomous vehicle, high-resolution multi beam sonar and wireless transmission module.([4])

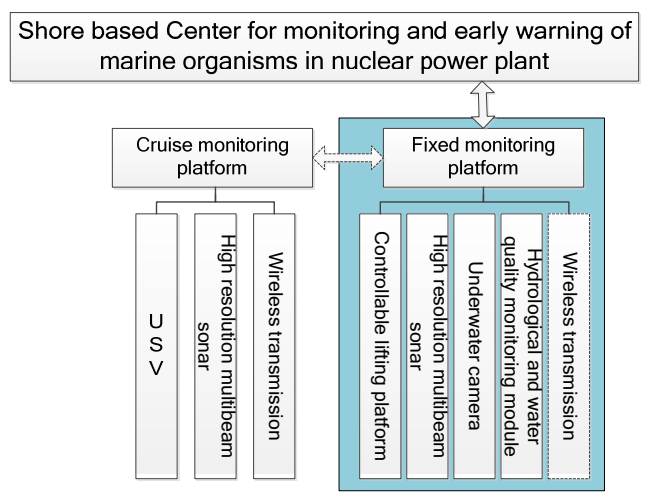

Fig.1. Test pond Block diagram of autonomous monitoring and early warning system for marine organisms in nuclear power plant

The data flow of the system is shown in Figure 2. At the front end of the detection, the acoustic signal is converted into electrical signal by the receiving sensor array, and xtf type three-dimensional acoustic image data and JPG format image file are directly processed at the pre-processing end. In the post-processing process, the image data is exported to the txt document or mat type document used by the algorithm program.([5])Finally, the density distribution is compared with the alarm threshold, the high and low levels are output, and the alarm device is controlled to start. In the initial stage of the project, the post-processing is carried out by manual processing. In the follow-up development of the project, professional software developers will be used to design the system architecture and realize the program of automatic processing.([6],[7])

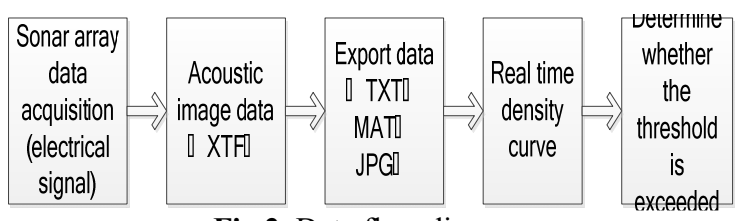

Fig.2. Data flow diagram

The input and output of the system after modularization. When the marine life automatic monitoring and early warning system is embedded into other parent systems as a subsystem, the input and output parameters of the subsystem are as follows: the input parameters include high frequency multi beam data (xtf format) collected by sonar array, GPS positioning data and control instructions; Taking jellyfish as an example, the output parameters include real-time (or quasi realtime) acoustic image data (jpg format), jellyfish density, maximum jellyfish size and maximum jellyfish echo intensity. As shown in Figure 3:

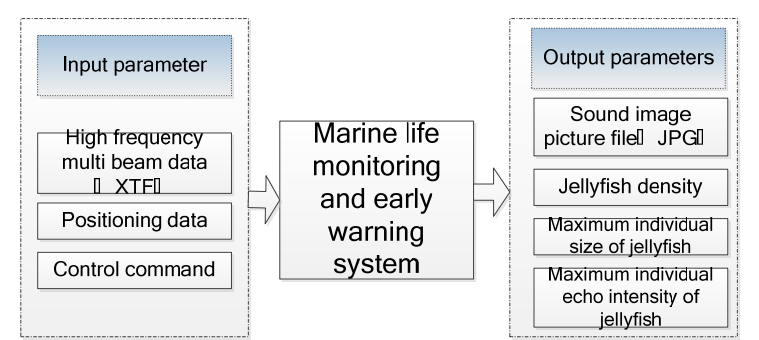

Fig.3. Input and output parameters of monitoring system after modularization

\subsection{Design of fixed marine life monitoring and early warning network}

Based on the high-resolution multi beam acoustic method to detect marine life, the marine life early warning system is constructed, as shown in Figure 4. At a certain distance from the cold source water intake of the power station, the monitoring line is established, the data link between the monitoring sonar and the communication buoy is established, and the data of the monitoring sonar is transmitted back to the shore monitoring room by the communication buoy.

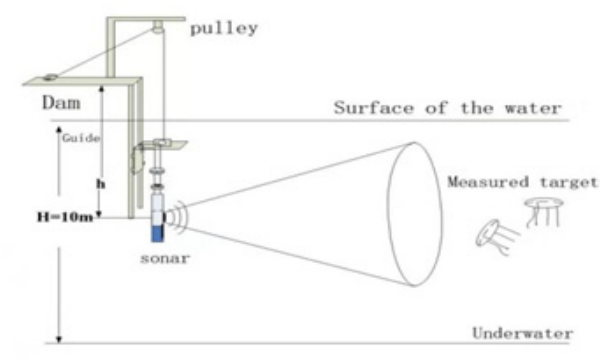

Fig.4. Working mode diagram of fixed monitoring node

\subsection{Mobile monitoring and early warning scheme based on USV}

USV (unmanned surface vehicle) is a low-cost and highefficiency small surface self-propelled vehicle. Based on the USV platform, the sonar is placed at a depth of about $1 \mathrm{~m}$ below the sea surface, and the acoustic beam is emitted obliquely downward to detect the jellyfish and other marine biological targets within the range of the sonar beam. The test system consists of USV, detection sonar, GPS positioning, wireless data transmission and shore control, as shown in Figure 5. 


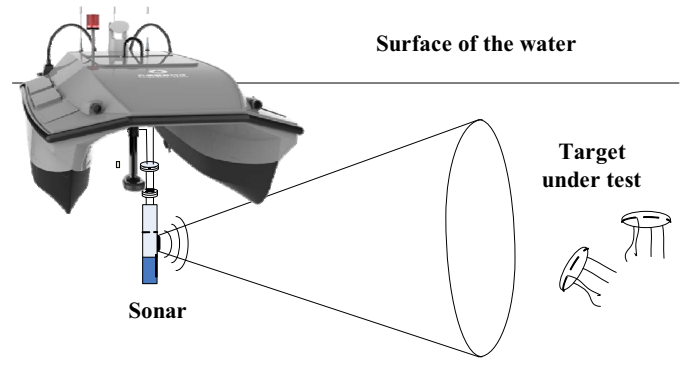

Fig.5.Schematic diagram of working mode based on USV

Different from the fixed monitoring platform, the mobile monitoring and early warning system based on USV makes full use of the mobility of USV platform, and can monitor the distribution of marine organisms on a large scale in cold source waters. In order to effectively monitor the distribution of marine organisms in the whole cold source sea area, it is necessary to reasonably design the mobile lines of USV platform, which must fully consider the specific conditions such as ocean current, sea terrain and coastline.

\section{Research on marine life self monitorin $\mathrm{g}$ and early warning technology}

\subsection{Investigation on species, characteristics and habits of marine organisms in nuclear power plant}

According to the types, characteristics and habits (hazard time period) of the harmful marine organisms in the sea area around the nuclear power plant, the systematic investigation and hazard assessment are carried out, and the knowledge base of the habits of the harmful marine organisms in nuclear power plant is established, so as to provide a priori perception information for the monitoring and early warning of the harmful marine organisms in nuclear power plant.

\subsection{Study on the monitoring methods of typical marine organisms affecting nuclear power safety}

Through the long-term detection of typical marine organisms individuals and clusters that affect the safety of nuclear power, the echo data of typical marine organisms are obtained, and the acoustic characteristics (relative echo intensity, acoustic scale, etc.) of typical marine organisms are analyzed and extracted, Finally, the real-time monitoring of typical marine organisms can be realized.

\subsection{Research on the typical early warning mechanism of marine organisms affecting nuclear power safety}

Using high-resolution multi beam sonar as sensing means, the echo data of marine organisms are analyzed, processed and identified by using the feature analysis and statistics module, and the density distribution of jellyfish, hairy shrimp and other marine organisms in the monitored sea area is obtained in real time. Combined with the threat degree of marine organisms invasion to the operation of nuclear power plant in the data information database, the alarm threshold is set, Real time warning of harmful marine organisms beyond the warning threshold.

\section{Research on system implementation a nd field monitoring scheme}

Based on the high-resolution multi beam acoustic detection technology of marine life, the marine life early warning system is constructed. It is proposed to arrange fixed monitoring stations on both sides of the water intake and at the center of the water intake, so as to solve the problem of rapid water area coverage monitoring capacity. Subsequently, the autonomous mobile monitoring platform will be built, the mobile route will be planned, and the whole area coverage monitoring of water intake width will be realized through cruise.

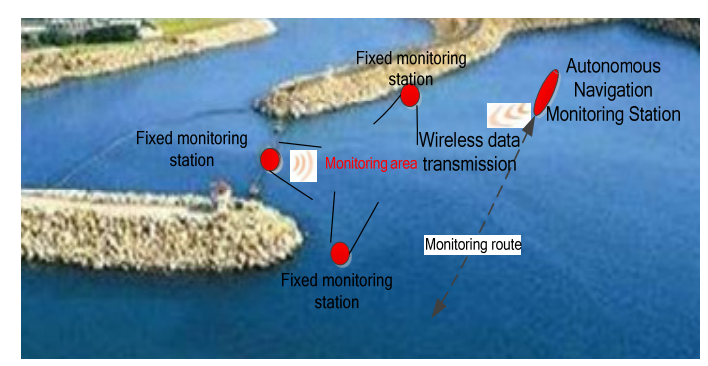

Fig.6.Layout of monitoring points of cold source water intake

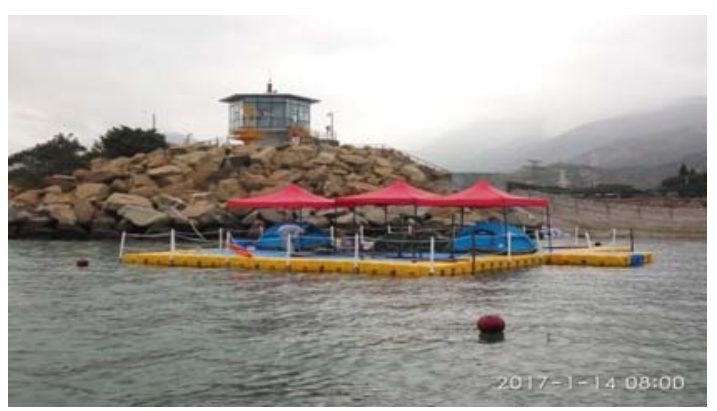

Fig.7. Test platform of a project

In the process of the research on the detection method of marine organisms based on the highresolution multi beam acoustic detection method, the acoustic characteristics of individual marine organisms such as jellyfish and fish swarm are analyzed and 
extracted, and the estimation method of the number and distribution density of marine organisms cluster is further studied.
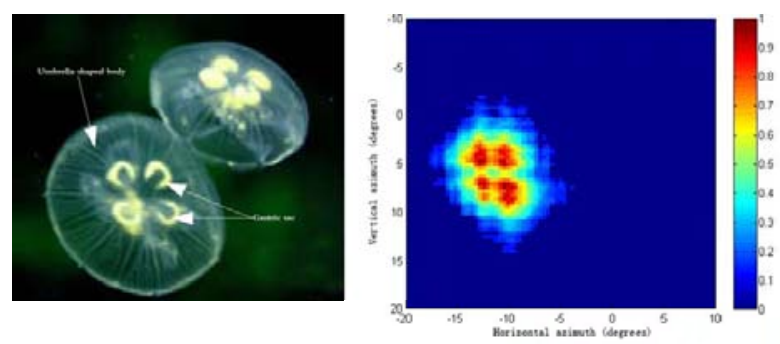

Fig.8.Real image and acoustic image of jellyfish
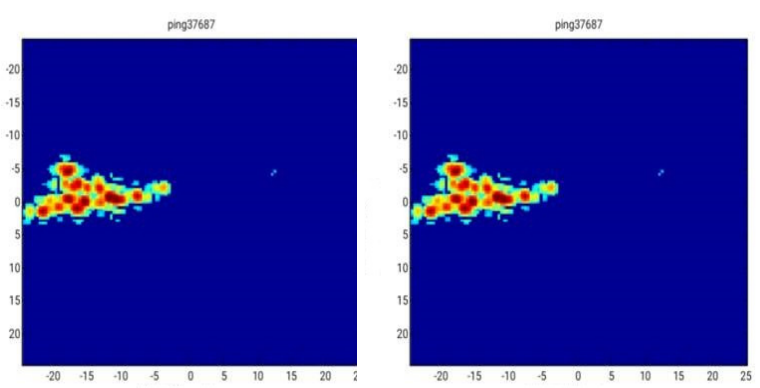

Fig.9. Fish echo image

Through the acoustic detection test of jellyfish and fish swarm in a certain project, we can clearly observe the echo image of jellyfish individual, the temporal spatial variation of jellyfish distribution density and the process of fish swarm appearing in the sonar field of vision, etc., which can provide technical support for the future research on monitoring and early warning technology of marine organisms harming nuclear power plants.

\section{Conclusion}

Based on the design and analysis of the detection and early warning scheme for harmful marine organisms at the water intake of nuclear power plant based on highresolution multi beam acoustic detection method, an automatic monitoring and early warning system for marine organisms is constructed, and the image information of marine organisms obtained is analyzed, processed and identified by using the feature analysis and statistics module with high-resolution multi beam sonar as sensing means, The density distribution of jellyfish, fish and other marine organisms in the monitoring area is obtained in real time, and the alarm is given when the threshold is exceeded, which verifies the technical feasibility.

Therefore, in order to avoid the threat of jellyfish, fish and other marine organisms attacking the water intake of nuclear power plant and improve the reliability of cold source sea area, it is necessary to establish a defense in depth system of marine organisms identification, detection, early warning and hierarchical response, in which detection and early warning is the front-end disposal measure, providing accurate and timely marine organisms information for subsequent hierarchical response, and can replace the underwater inspection work of divers, Reduce the risk of industrial safety.

\section{References}

1. Fischell EM, Schmidt $\mathrm{H}$ Classification of underwater targets from autonomous underwater vehicle sampled bistatic acoustic scattered fields. J Acoust Soc Am 138(6) 357: (2015)

2. Morse SF, Marston PL, Kaduchak G Highfrequency backscattering enhancements by thick finite cylindrical shells in water at oblique incidence: experiments, interpretation, and calculations. J Acoust Soc Am 103(2):785-794 (1998).

3. Ristic, B., Vo, B.N., Clark, D. and Vo, B.T.. A metric for performance evaluation of multi-target tracking algorithms, IEEE Transactions on Signal Processing, 59(7), 3452-3457(2011).

4. Raj, K.D. and Krishna, I.M. Kalman filter based target tracking for track while scan data processing, Proceeedings of the 2nd International Conference on Electronics and Communication Systems (ICECS), IEEE, Coimbatore, India, pp. 878-883(2015).

5. Tang, X., Tharmarasa, R., McDonald, M. and Kirubarajan, T.. Multiple detection-aided lowobservable track initialization using ML-PDA, IEEE Transactions on Aerospace and Electronic Systems, 53(2), 722-735(2017).

6. Li XK, Meng XX, Xia Z. Characteristics of the geometrical scattering waves from underwater target in fractional Fourier transform domain . Acta Physica Sinica, 64(2015).

7. Fan J, Zhu BL, Tang WL. Modified geometrical highlight model of echoes from nonrigid surface sonar target. Aeta Acustica, 26(6), 545 (2001). 